\title{
Molecular characterization of Staphylococcus aureus isolates causing skin and soft tissue infections (SSTIs)
}

\author{
Dan Yao+1, Fang-you Yu², Zhi-qiang Qin³, Chun Chen'1, Su-su He1, Zeng-qiang Chen², Xue-qing Zhang² and Liang- \\ xing Wang*1
}

\begin{abstract}
Background: Staphylococcus aureus, particularly methicillin-resistant S. aureus (MRSA), is an important cause of pyogenic skin and soft tissue infections (SSTIS). The aim of present study is to investigate the molecular characteristic of Staphylococcus aureus isolates isolated from the pus samples from the patients with purulent skin and soft tissue infections in Wenzhou, China.

Methods: Between December 2002 and June 2008, a total of 111 nonduplicate S. aureus isolates were collected from the pus samples of the patients with SSTIs in a teaching hospital in Wenzhou, China. All the tested isolates were confirmed as S. aureus using a Staph SPA agglutination kit, Gram's stain and a Vitek-60 microbiology analyzer. The homology among the tested isolates was determined by pulsed-field gel electrophoresis (PFGE). Multilocus sequence typing (MLST) was used to determine the sequence types (STs) of the selected isolates. The genotypes of SCCmec were determined by a multiplex PCR in the MRSA isolates. Panton-Valentine leukocidin (PVL) genes and mecA were also determined by another multiplex PCR.
\end{abstract}

Results: Among the $111 \mathrm{~S}$. aureus isolates, 48 and 63 isolates were community-acquired and hospital-acquired respectively. Sixty isolates were confirmed as MRSA harboring mecA detected by PCR. A total of 32 PFGE clonal types were obtained by PFGE, with 10 predominant patterns (types A to J). Twenty-five different STs including ST398 and three novel STs were found among 51 selected isolates. The main STs were ST239, ST1018, ST59, ST7 and ST88. Of 60 MRSA isolates, SCCmec II, III, IV and SCCmec V were found in three, 50, three and two isolates, respectively. The positive rates of PVL genes in overall isolates, HA-isolates, CA-isolates, MRSA isolates and MSSA isolates were $23.4 \%(26 / 111)$, $20.6 \%$ (13/63), 27.1\% (13/48), 21.7\% (13/60) and 25.5\% (13/51), respectively. Eight (33.3\%, 8/24) of 24 CA-MRSA isolates and $5(13.9 \%, 5 / 36)$ of 36 HA-MRSA isolates were positive for PVL genes. ST239-MRSA-SCCmeclll and ST1018-MRSASCCmeclll clones were found to be main clones and spread between community and hospital.

Conclusion: S. aureus isolates causing SSTIs showed considerable molecular heterogeneity and harbored high prevalence of PVL genes. Clonal spread was responsible for the dissemination of the isolates of S. aureus associated with SSTIS.

\section{Background}

Infections caused by Staphylococcus aureus, especially methicillin-resistant S. aureus (MRSA), are emerging as a major public health problem. MRSA has been associated with skin and soft tissue infections (SSTIs), endovascular

\footnotetext{
*Correspondence: wzyxywlx@163.com

1 Department of Respiratory Medicine, the First Affiliated Hospital of Wenzhou Medical College, Wenzhou325000, China

+ Contributed equally

Full list of author information is available at the end of the article
}

infections, pneumonia, septic arthritides, endocarditis, osteomyelitis and sepsis [1]. Since the first European isolate of MRSA was detected in 1960s, MRSA has become a leading cause of nosocomial infections worldwide [1,2]. In recent years, making even more complex the understanding of the epidemiology is the widespread dissemination of community-associated MRSA (CA-MRSA) afflicting individuals with no apparent risk factors for hospital acquisition and often possessing a potential viru- 
lence factor, Panton-Valentine leukocidin (PVL) [2]. The carriage of PVL genes, pulsed-field gel electrophoresis (PFGE), staphylococcal cassette chromosome mec (SCCmec) typing and multilocus sequence typing (MLST) were performed to monitor the evolutionary process of pandemic clones [3-6]. However, PVL genes have been detected infrequently in hospital-acquired MRSA isolates (HA-MRSA) in the Western Europe [5,7,8]. During the last decade, several major pandemic clones have been identified, including Brazilian clone, Hungarian clone, Portuguese clone, New York/Japan clone, Pediatric clone, Iberian clone and UK EMRSA-16 [9]. In China, the data from previous studies showed that the widespread predominant clone among HA-MRSA isolates was the Hungarian clone (ST239 SCCmec III) [10,11]. However, the epidemiology of Chinese $S$. aureus isolates isolated from the patients with SSTIs is poorly documented. The aim of this study was to investigate the epidemiology of $S$. aureus isolates associated with SSTIs by a variety of molecular methods and epidemiological data in a teaching hospital in Wenzhou, China.

\section{Methods}

From December 2002 to June 2008, a total of 111 nonduplicate $S$. aureus isolates were collected from the pus samples of the patients with SSTIs including lesions requiring incision and drainage or with spontaneously draining purulent fluid, carbuncles, furuncles, boils, cellulitis with purulent drainage, chronic ulcer and deep wounds in a teaching hospital in Wenzhou, China. The $S$. aureus isolates isolated from the patients with SSTIs with clinical signs and symptoms of infection including increased white blood cell counts, fevers, redness, swelling, and exudate, were considered for inclusion. The isolates isolated from the patients without clinical signs and symptoms of infection were considered for colonization and exclusion. The clinical isolates were confirmed as $S$. aureus using a Staph SPA agglutination kit (bioMe'rieux, Marcy l'Etoile, France), Gram's stain and a Vitek-60 microbiology analyser (bioMe'rieu, Marcy l' Etoile, France). The isolates, which were isolated from the patients who had no risk factors for nosocomial acquisition and no hospitalizations or nursing home residence within a year before hospital admission within 48 hours after hospital admission, were considered to be community-associated. The isolates, which were isolated from the inpatients with no infections before hospital admission and more than 48 hours after hospital admission, were determined as HA-isolates. All tested isolates were digested with lysostaphin $(1 \mathrm{mg} / \mathrm{ml})$ (Sangon, China) at $37^{\circ} \mathrm{C}$ for $30 \mathrm{~min}$. All the DNA was purified following the instruction of the Genomic DNA Extraction kit (Sangon, China). The DNA was stored at $-20^{\circ} \mathrm{C}$ and prepared for PCR detection. Chromosomal DNA was prepared from all the isolates and cleaved with $40 \mathrm{U} X b a \mathrm{I}$ (Sangon, China). Electrophoresis was performed on $1 \%$ agarose gels in $0.5 \mathrm{M}$ Tris/borate/EDTA buffer on a CHEF-Mapper XA PFGE system (Bio-Rad, Hercules, CA) for 22 hours at $14^{\circ} \mathrm{C}$, with run conditions of $6 \mathrm{~V} / \mathrm{cm}$, a pulse angle of 120 degrees and pulse times from 5 to $20 \mathrm{sec}-$ onds. A $\lambda$ DNA ladder (Amersham Biosciences, Piscataway, NJ) was used as molecular mass marker and bands were stained with ethidium bromide $(0.5 \mu \mathrm{g} / \mathrm{ml})$ and photographed under UV light. PFGE profiles were interpreted by the criteria described previously [12]. MLST was performed as described previously and ST numbers were assigned according to the S. aureus database http:// saureus.mlst.net/[3]. The SCCmec typing of MRSA isolates was performed under the conditions as described previously [6]. The SCCmec V isolates were further determined by the methods described by Zhang et al and Milheirico et al. [6,13]. Reference strains were MRSA NCTC 10442 (SCCmec I), MRSA N315 (SCCmec II), MRSA 85/ 2082 (SCCmec III), MRSA JCSC 4744 (SCCmec IV) and MRSA HS663 (SCCmec V). The presences of the mec A and PVL genes were determined as described previously [5].

\section{Ethical approval was not needed for the present study}

\section{Results}

One hundred and eleven $S$. aureus isolates were isolated from the patients aged from 4 days to 80 years old with purulent SSTIs. Among the 111 S. aureus isolates, 48 CAisolates were associated generally with the diseases of purulent dermatitis, burns, pemphigus, maternal breast abscesses, while 63 HA-isolates were mainly associated with surgical intervention, burns or other localized infections with the risk factors as follows: the histories of prior hospitalization in an acute-care setting, hemodialysis, enter via-catheterization, arteriovenous shunts, tracheostomy or surgery in the year preceding MRSA isolation. And all the patients with purulent SSTIs caused by $S$. aureus recovered under the treatment of antibiotic and appropriate nursing care. Sixty isolates were confirmed as MRSA determined by PCR for detecting mecA. Of 60 MRSA isolates, 36 and 24 isolates were hospital-associated and community-associated.

The 111 isolates were clustered into 32 PFGE clonal types by PFGE, with 10 predominant patterns (designated types A to J) accounting for 72 isolates. The most prevalent PFGE clonal type was type A (13 isolates). The isolates belonging to PFGE type E, $\mathrm{H}, \mathrm{I}$ and $\mathrm{J}$ were found only in HA-isolates, while the isolates belonging to PFGE type A, B, C, D, F, and G were detected not only in HAisolates but also in CA-isolates.

MLST was performed on representative isolates based on PFGE type and all PVL-positive isolates. A total of 25 
different sequence types (STs) were found among 51 selected isolates, mainly including ST239, ST1018, ST88, ST59 and ST7 (Table. 1). Three novel STs including ST1349, ST1357 and ST1358, were first found in the present study. Twenty-one STs including novel ST1349 and ST1358 were found in CA-isolates, while 17 STs were found in HA-isolates. The most dominant ST was ST239 which was found in $20 \mathrm{HA}$-isolates and four CA-isolates. Twenty ST239 HA-isolates persisting in our hospital from 2003 to 2008 were found among PFGE types B, E, J and $\mathrm{M}$ isolates and four ST239 CA-isolates were detected among PFGE type B isolates in 2007. ST1018 was found to be the second common ST. Seven ST1018 CA-isolates and six ST1018 HA-isolates were found between 2003 and 2008. The ST of six HA-isolates and four CA-isolates was ST88. Nine of 11 ST59 isolates were HA-isolates isolates. ST 88 was the most common ST in the PVL-positive isolates $(7 / 26)$.

Of 60 MRSA isolates, three, 50, three and two isolates harbored SCCmec II, SCCmec III, SCCmec IV and SCC$m e c \mathrm{~V}$, respectively. The remaining two isolates were nontypeable by the multiplex PCR method used.

Twenty-six isolates were positive for PVL genes. The positive rates of PVL genes in overall isolates, HA-isolates, CA-isolates, MRSA isolates and MSSA isolates were $23.4 \%$ (26/111), 20.6\% (13/63), 27.1\% (13/48), 21.7\% $(13 / 60)$ and $25.5 \%(13 / 51)$, respectively. The prevalence of

Table 1: Molecular characterization of 111 isolates.

SCCmec type

\begin{tabular}{|c|c|c|c|c|c|c|c|c|c|c|c|c|}
\hline ST & $\begin{array}{c}\text { No. } \\
\text { of isolates }\end{array}$ & PFGE & $\begin{array}{c}\text { No. } \\
\text { of MRSA }\end{array}$ & $\begin{array}{c}\text { No. of } \\
\text { PVL-positive } \\
\text { strain }\end{array}$ & $\begin{array}{c}\text { No. of } \\
\text { CA-isolates }\end{array}$ & I & & II & II & & & NT \\
\hline
\end{tabular}

\begin{tabular}{|c|c|c|c|c|c|c|c|c|c|c|}
\hline 239 & 24 & $B, E, J, M$ & 19 & 4 & 4 & & 19 & & & \\
\hline 1018 & 13 & $A$ & 10 & 2 & 7 & 1 & 9 & & & \\
\hline 59 & 11 & $\mathrm{D}, \mathrm{H}$ & 2 & 0 & 2 & & 2 & & & \\
\hline 7 & 10 & $C$ & 2 & 2 & 3 & & 2 & & & \\
\hline 88 & 10 & $\mathrm{G}, \mathrm{I}$ & 6 & 6 & 4 & & 3 & 3 & & \\
\hline 188 & 6 & $\mathrm{~F}$ & 1 & 0 & 4 & & 1 & & & \\
\hline 5 & 4 & $K, X$ & 2 & 2 & 3 & 2 & & & & \\
\hline 25 & 3 & $\mathrm{~N}$ & 2 & 2 & 3 & & 1 & & 1 & \\
\hline 243 & 3 & $\mathrm{O}$ & 2 & 0 & 2 & & 1 & & & 1 \\
\hline 594 & 3 & L & 3 & 0 & 2 & & 2 & & 1 & \\
\hline 72 & 2 & $\mathrm{R}$ & 1 & 1 & 0 & & 1 & & & \\
\hline 366 & 2 & $S$ & 2 & 0 & 2 & & 2 & & & \\
\hline 398 & 2 & NT & 1 & 2 & 1 & & 1 & & & \\
\hline 837 & 2 & $O A, O D$ & 1 & 0 & 2 & & 0 & & & 1 \\
\hline 944 & 2 & $\mathrm{Q}$ & 1 & 0 & 1 & & 1 & & & \\
\hline 1301 & 2 & $P$ & 0 & 2 & 0 & & & & & \\
\hline 86 & 2 & $\mathrm{~V}, \mathrm{~W}$ & 1 & 1 & 1 & & 1 & & & \\
\hline 1 & 2 & U & 0 & 0 & 2 & & & & & \\
\hline 15 & 2 & $\mathrm{~T}$ & 0 & 0 & 1 & & & & & \\
\hline 20 & 1 & Y & 1 & 0 & 1 & & 1 & & & \\
\hline 370 & 1 & Z & 0 & 0 & 0 & & & & & \\
\hline 1019 & 1 & $\mathrm{OB}$ & 1 & 0 & 1 & & 1 & & & \\
\hline 1349 & 1 & OC & 1 & 1 & 1 & & 1 & & & \\
\hline 1357 & 1 & $\mathrm{OE}$ & 1 & 1 & 0 & & 1 & & & \\
\hline 1358 & 1 & OF & 0 & 0 & 1 & & & & & \\
\hline
\end{tabular}


PVL genes among CA-MRSA isolates $(33.3 \%, 8 / 24)$ was higher than that among HA-MRSA isolates $(13.9 \%, 5 / 36)$.

On the basis of MLST and SCCmec typing, ST239MRSA-SCCmec III clone was found to be the most prevalent clone among HA-isolates (19/63), while ST1018MRSA-SCCmec III clone was more likely to be obtained among CA-isolates (7/48). ST239-MRSA- SCCmec III and ST1018-MRSA- SCCmec III clones were obtained both in CA-isolates and HA-isolates. ST88-MRSA-SCC$m e c$ IV clone was found in three HA-isolates. The ST of the two isolates which could not be digested with SmaI was documented as ST398. One ST398- CA-MRSASCCmec III isolate was detected from a patient with the infection of gluteal muscles in 2002. The other ST398 isolate, which was detected in a patient aged 48 with the surgery of renal transplantation, was HA-MSSA. Novel ST1349, ST1357 and ST1358 were found in one PVLpositive CA-MRSA-SCCmec III isolate, one PVL-positive HA-MRSA-SCCmec III isolate and one CA-MSSA isolate, respectively.

Two MRSA isolates harbor SCCmec V were community-associated.

\section{Discussion}

PFGE type A, B, C, D, F, G and $\mathrm{K}$ isolates had penetrated both in hospital and community in specified years, indicating that $S$. aureus isolates associated with SSTIs were spreading between community and hospital in Wenzhou, China. Whereas PFGE type E isolates were spread only in hospital. Previous studies demonstrated that the most prevalent clones in China were Brazilian/Hungarian (ST239-MRSA- SCCmec III) and New York/Japan (ST5MRSA-SCCmec II) clones and had unique geographic distributions $[10,11,14]$. Similar to previous studies, ST239-MRSA-SCCmec III clone was also found to be the most prevalent clone among $S$. aureus isolates associated with SSTIs both in community and hospital. In contrast to previous study, only two ST5-MRSA- SCCmec II isolates were found in the present study. Although ST1018MRSA-SCCmecIII clone was not widely reported before, the clone was predominantly detected among CA $S$. aureus isolates, ST88 was the most prevalent ST among PVL-positive S. aureus isolates in our previous study [15]. ST88 clone was also an important clone among $S$. aureus isolates causing SSTIs. We had reported the molecular characteristic of $10 \mathrm{PVL}$-positive S. aureus isolates associated with SSTIs from January 2005 to January 2006 [15]. The data of the present study included the six PVL-positive isolates associated with SSTIs in our previous study [15]. Because other four PVL-positive MRSA isolates including one ST398 isolate died, the data of these four isolates were excluded from the present study. Historically it has been reported that HA-MRSA isolates mainly harbor SCCmec I, II and III, whereas SCCmec IV and V are found mainly in CA-MRSA isolates [16,17].

In contrast to previous studies, SCCmec IV was found in HA-MRSA isolates and SCCmec III was found both in HA- and CA-MRSA isolates, indicating that MRSA isolates harboring SCCmec IV and MRSA isolates harboring SCCmec III can spread between hospital and community.

PVL was supposed to be an important virulent factor in CA-MRSA isolates and a strong epidemiological association between PVL genes and successful CA-MRSA lineages, especially with the disease of SSTIs [18-20]. We also found the high prevalence of PVL genes among both HA- and CA-isolates and that the prevalence of PVL genes in HA-isolates was almost as high as that in CAisolates. However, there was higher prevalence of PVL genes in CA-MRSA isolates than that in HA-MRSA isolates. MRSA clonal type ST398 is usually associated with the infections of animals, especially pigs [21,22]. However, human infections caused by MRSA ST398 isolates have been reported in many countries [23,24]. PVL-positive MRSA ST398 isolates associated with human infections has been reported [15,25]. Two PVL positive $S$. aureus ST398 isolates including one MRSA isolate and one MSSA isolate were also found in the present study. These data indicate that immediate monitoring is required for the spread of MRSA ST398 isolates.

\section{Conclusion}

S. aureus isolates causing SSTIs showed considerable molecular heterogeneity and harbored high prevalence of PVL genes. Clonal spread was responsible for the dissemination of the isolates of $S$. aureus associated with SSTIs.

\section{Competing interests}

The authors declare that they have no competing interests.

\section{Authors' contributions}

DY, SSH and CC performed the laboratory measurements. FYY and LXW made substantial contributions to conception and design, and revised the manuscript critically for important intellectual content. ZQQ performed the analysis and interpretation of data. ZQC, XQZ and CC participated in design and coordination. DY and FYY drafted the manuscript. All authors read and approved the final manuscript.

\section{Acknowledgements}

We gratefully acknowledge Professor Minggui Wang, Hua Shan Affiliated Hos pital of Shanghai Medical School of Fudan University for providing the technique of pulsed-field gel electrophoresis. And we are also grateful to T. Ito for the kind gift of MRSA NCTC 10442 (SCCmec I), MRSA N315 (SCCmec II), MRSA85/2082 (SCCmec III), and MRSA JCSC4744 (SCCmec IV). This study was supported by a grant from national natural science foundation of China (No.30970124)

\section{Author Details}

1Department of Respiratory Medicine, the First Affiliated Hospital of Wenzhou Medical College, Wenzhou325000, China, ${ }^{2}$ Department of Laboratory Medicine, the First Affiliated Hospital of Wenzhou Medical College, Wenzhou.325000, China and 3Division of Infectious Diseases, Department of Medicine, Hollings Cancer Center, Medical University of South Carolina, South Carolina, USA 
Received: 28 September 2009 Accepted: 26 May 2010

Published: 26 May 2010

\section{References}

1. Lowy FD: Staphylococcus aureus infections. N Engl J Med 1998, 339(8):520-532.

2. Tristan A, Bes M, Meugnier H, Lina G, Bozdogan B, Courvalin P, Reverdy ME, Enright MC, Vandenesch F, Etienne J: Global distribution of PantonValentine leukocidin--positive methicillin-resistant Staphylococcus aureus. Emerg Infect Dis 2007, 13(4):594-600.

3. Enright MC, Day NP, Davies CE, Peacock SJ, Spratt BG: Multilocus sequence typing for characterization of methicillin-resistant and methicillin-susceptible clones of Staphylococcus aureus, 2006. J Clin Microbiol 2000, 38(3):1008-1015.

4. Bannerman TL, Hancock GA, Tenover FC, Miller JM: Pulsed-field gel electrophoresis as a replacement for bacteriophage typing of Staphylococcus aureus. J Clin Microbiol 1995, 33(3):551-555.

5. McClure JA, Conly JM, Lau V, Elsayed S, Louie T, Hutchins W, Zhang K: Novel multiplex PCR assay for detection of the staphylococcal virulence marker Panton-Valentine leukocidin genes and simultaneous discrimination of methicillin-susceptible from resistant staphylococci. J Clin Microbiol 2006, 44(3):1141-1144.

6. Zhang K, McClure JA, Elsayed S, Louie T, Conly JM: Novel multiplex PCR assay for characterization and concomitant subtyping of staphylococcal cassette chromosome mec types I to V in methicillinresistant Staphylococcus aureus. J Clin Microbio/ 2005, 43(10):5026-5033.

7. Goering RV, Shawar RM, Scangarella NE, O'Hara FP, Amrine-Madsen H, West JM, Dalessandro M, Becker JA, Walsh SL, Miller LA, et al:: Molecular epidemiology of methicillin-resistant and methicillin-susceptible Staphylococcus aureus isolates from global clinical trials. J Clin Microbiol 2008, 46(9):2842-2847.

8. Lina G, Piemont Y, Godail-Gamot F, Bes M, Peter MO, Gauduchon V, Vandenesch F, Etienne J: Involvement of Panton-Valentine leukocidinproducing Staphylococcus aureus in primary skin infections and pneumonia. Clin Infect Dis 1999, 29(5):1128-1132.

9. Oliveira DC, Tomasz A, de Lencastre H: Secrets of success of a human pathogen: molecular evolution of pandemic clones of meticillinresistant Staphylococcus aureus. Lancet Infect Dis 2002, 2(3):180-189.

10. Xu BL, Zhang G, Ye HF, Feil EJ, Chen GR, Zhou XM, Zhan XM, Chen SM, Pan WB: Predominance of the Hungarian clone (ST 239-III) among hospitalacquired meticillin-resistant Staphylococcus aureus isolates recovered throughout mainland China. J Hosp Infect 2009, 71(3):245-255.

11. Zhang W, Shen X, Zhang H, Wang C, Deng Q, Liu L, Yang Y: Molecular epidemiological analysis of methicillin-resistant Staphylococcus aureus isolates from Chinese pediatric patients. Eur J Clin Microbiol Infect Dis 2009, 28(7):861-4.

12. Tenover FC, Arbeit RD, Goering RV, Mickelsen PA, Murray BE, Persing DH, Swaminathan B: Interpreting chromosomal DNA restriction patterns produced by pulsed-field gel electrophoresis: criteria for bacterial strain typing. J Clin Microbiol 1995, 33(9):2233-2239.

13. Milheirico C, Oliveira DC, de Lencastre H: Update to the multiplex PCR strategy for assignment of mec element types in Staphylococcus aureus. Antimicrob Agents Chemother 2007, 51(9):3374-3377.

14. Liu Y, Wang H, Du N, Shen E, Chen H, Niu J, Ye H, Chen M: Molecular evidence for spread of two major methicillin-resistant Staphylococcus aureus clones with a unique geographic distribution in Chinese hospitals. Antimicrob Agents Chemother 2009, 53(2):512-518.

15. Yu F, Chen Z, Liu C, Zhang X, Lin X, Chi S, Zhou T, Chen X: Prevalence of Staphylococcus aureus carrying Panton-Valentine leukocidin genes among isolates from hospitalised patients in China. Clin Microbiol Infect 2008, 14(4):381-384

16. Ito T, Ma XX, Takeuchi F, Okuma K, Yuzawa H, Hiramatsu K: Novel type V staphylococcal cassette chromosome mec driven by a novel cassette chromosome recombinase, ccrC. Antimicrob Agents Chemother 2004 48(7):2637-2651.

17. Ma XX, Ito T, Tiensasitorn $C$, Jamklang M, Chongtrakool P, Boyle-Vavra S, Daum RS, Hiramatsu K: Novel type of staphylococcal cassette chromosome mec identified in community-acquired methicillinresistant Staphylococcus aureus strains. Antimicrob Agents Chemother 2002, 46(4):1147-1152.
18. Naas T, Fortineau N, Spicq C, Robert J, Jarlier V, Nordmann P: Three-year survey of community-acquired methicillin-resistant Staphylococcus aureus producing Panton-Valentine leukocidin in a French university hospital. J Hosp Infect 2005, 61(4):321-329.

19. Chini V, Petinaki E, Foka A, Paratiras S, Dimitracopoulos G, Spiliopoulou I: Spread of Staphylococcus aureus clinical isolates carrying PantonValentine leukocidin genes during a 3-year period in Greece. Clin Microbiol Infect 2006, 12(1):29-34.

20. Diep BA, Sensabaugh GF, Somboona NS, Carleton HA, PerdreauRemington F: Widespread skin and soft-tissue infections due to two methicillin-resistant Staphylococcus aureus strains harboring the genes for Panton-Valentine leukocidin. J Clin Microbiol 2004 42(5):2080-2084

21. Smith TC, Male MJ, Harper AL, Kroeger JS, Tinkler GP, Moritz ED, Capuano AW, Herwaldt LA, Diekema DJ: Methicillin-resistant Staphylococcus aureus (MRSA) strain ST398 is present in midwestern U.S. swine and swine workers. PLOS ONE 2008, 4(1):e4258.

22. van Duijkeren E, Ikawaty $R$, Broekhuizen-Stins MJ, Jansen MD, Spalburg EC, de Neeling AJ, Allaart JG, van Nes A, Wagenaar JA, Fluit AC: Transmission of methicillin-resistant Staphylococcus aureus strains between different kinds of pig farms. Vet Microbiol 2008, 126(4):383-389.

23. Pan A, Battisti A, Zoncada A, Bernieri F, Boldini M, Franco A, Giorgi M, lurescia M, Lorenzotti S, Martinotti M, et al:: Community-acquired methicillin-resistant Staphylococcus aureus ST398 infection, Italy. Emerg Infect Dis 2009, 15(5):845-847.

24. Krziwanek K, Metz-Gercek S, Mittermayer H: Methicillin-Resistant Staphylococcus aureus ST398 from human patients, upper Austria. Emerg Infect Dis 2009, 15(5):766-769.

25. Christina Welinder-Olsson, Kerstin Florén-Johansson, Larsson Leif, Sven Öberg, Karlsson Lisbeth, Christina Åhrén: Infection with PantonValentine Leukocidin-Positive Methicillin-Resistant Staphylococcus aureus t034. Emerg Infect Dis 2008, 14(8):1271-1272.

\section{Pre-publication history}

The pre-publication history for this paper can be accessed here: http://www.biomedcentral.com/1471-2334/10/133/prepub

doi: 10.1186/1471-2334-10-133

Cite this article as: Yao et al., Molecular characterization of Staphylococcus aureus isolates causing skin and soft tissue infections (SSTIs) BMC Infectious Diseases 2010, 10:133

\section{Submit your next manuscript to BioMed Centra and take full advantage of:}

- Convenient online submission

- Thorough peer review

- No space constraints or color figure charges

- Immediate publication on acceptance

- Inclusion in PubMed, CAS, Scopus and Google Scholar

- Research which is freely available for redistribution
C Biomed Central 